\title{
Performance study of dimensionality reduction methods for metrology of nonrigid mechanical parts
}

\author{
H. Radvar-Esfahlan* and S.-A. Tahan \\ Laboratoire d'Ingénierie des Produits, Procédés et Systèmes (LIPPS), École de Technologie Supérieure, Montreal, \\ H3C 1K3, Canada
}

Received: 29 June 2013 / Accepted: 20 October 2013

\begin{abstract}
The geometric measurement of parts using a coordinate measuring machine (CMM) has been generally adapted to the advanced automotive and aerospace industries. However, for the geometric inspection of deformable free-form parts, special inspection fixtures, in combination with CMM's and/or optical data acquisition devices (scanners), are used. As a result, the geometric inspection of flexible parts is a consuming process in terms of time and money. The general procedure to eliminate the use of inspection fixtures based on distance preserving nonlinear dimensionality reduction (NLDR) technique was developed in our previous works. We sought out geometric properties that are invariant to inelastic deformations. In this paper we will only present a systematic comparison of some well-known dimensionality reduction techniques in order to evaluate their accuracy and potential for non-rigid metrology. We will demonstrate that even though these techniques may provide acceptable results through artificial data on certain fields like pattern recognition and machine learning, this performance cannot be extended to all real engineering metrology problems where high accuracy is needed.
\end{abstract}

Keywords: Computer aided inspection; geometric inspection; flexible parts; dimensionality reduction

\section{Introduction}

Geometric inspection, geometric modeling, range data acquisition and analysis have developed as separate fields of engineering among the various engineering and scientific communities. However, all these fields share common scientific concepts, and there are many missed opportunities because of a lack of mutual connection and wasted synergy. Computer-Aided Inspection is one of these connection points, while nonrigid geometric inspection shares a profound degree of understanding of all the mentioned disciplines. Currently, a flexible workpiece must be constrained or clamped during the measurement process in order to simulate the use state. To that end, expensive and special inspection fixtures need to be designed and manufactured [1]. On the other hand, some inspection stages cannot be fully automated with this conventional approach. As a result, the geometric inspection of flexible parts remains a time and money consuming process. Typically some inspection set-up processes for nonrigid parts in aerospace industry request over $60 \mathrm{~h}$ of operations. On the other hand, even for simple parts, the quality of a planned inspection depends on the ability and experience of the operator. Despite the multitude of papers and research that have been produced in the CAD, CAM and CAI fields,

* Correspondence: hassan.radvar-esfahlan.1@ens.etsmtl.ca the inspection of flexible parts continues to pose difficulties and significant costs to industries because they need special fixation devices. This is also evidence of the lack of knowledge and theoretical foundations surrounding this special field. Our approach [2-4] was an effort to eliminate the use of special inspection fixtures in the metrology of flexible parts. We tried to provide a better understanding of the developed algorithms by having the comparison between different existing methods. We also added some techniques to robustify our Generalized Numerical Inspection Fixture (GNIF) [5]. Our philosophy was based on the fact that the interpoint shortest path (geodesic distance) between any two points on the parts remains unchanged during an isometric deformation. We called this property distance preserving property of nonrigid parts. In fact GNIF was inspired by a real industrial inspection process. When a flexible part is put on an inspection fixture the prevailing idea is that we are going to simulate the state of use. But more specifically one can say that we are looking for some correspondence between distorted parts and inspection fixtures, which represents our CAD-model. In spite of the accuracy of the presented methodology, the similarity detection process was extremely slow even for simple parts with zero Gaussian curvature. In this paper we will present a comparison of some well-known dimensionality reduction techniques in order to evaluate their accuracy and potential for non-rigid metrology. 
In Section 2 a brief introduction to six NLDR methods will be presented concisely with theirs mathematical fundaments. Then in Section 3 described methods will be evaluated using some typical world engineering data. The aim is to illustrate a systematic comparison and precision for each method.

\section{Dimensionality reduction}

Most problems in pattern recognition, such as image processing and speech recognition, begin with the preprocessing of high-dimensional signals. The complexity of most learning algorithms depends on the number of input dimensions $D$. This is why we are interested in reducing the embedding dimensionality with minimizing the loss of information, of course in the literature there are two techniques for dimensionality reduction: feature selection and feature extraction. In feature selection the aim is to find $d$ of $D$ dimensions (where $d<D$ ) which gives us the most information. In other words we are interested in finding the best subset of the set of the features. In metrology, feature selection is not a good approach for dimensionality reduction because the individual vertices do not carry much information on their own. It is the combination of vertices that provides the most discriminative information. This is the idea behind the feature extraction techniques. We therefore consider the following problem. Given a high dimensional data $X=\left(x_{1}, \ldots, x_{n}\right)$ where $x_{i} \in \mathbb{R}^{D}$ the aim is to compute the output data $Z_{i} \in \mathbb{R}^{d}$ that is the low dimensional representation of $X$. For techniques used in this paper only general information, including the steps for each method, will be included without going into derivation. Our focus in this paper is to compare the dimensionality reduction methods on the geometric metrology view point. Consequently, the aim is not to provide the details of the algorithms. We invite the reader to refer to the original paper of each algorithm for further details. However we will sketch a concise summary of each algorithm for comparison and reference purposes. Next section deals with methods that reduce the dimensionality of data by using distance and topology preservation as the criterion.

\subsection{Distance preserving DR techniques}

For linear dimensionality reduction, some simple criteria like maximizing the variance preservation leads to one of the robust dimensionality reduction methods like Principal Component Analysis (PCA) [6]. However in nonlinear cases the use of the same simple criteria requires more complex data models. On the other hand, every manifold can be described by its pairwise point distances whether by Euclidean, graph or geodesics metrics. Tons of research has been undertaken and motivated by a simple fact: if close points are kept close and far points kept far, then the high dimensional data set and its low dimensional embedding share the same shape [7]. This section attempts to review some of the best-known existing methods.

\subsubsection{Multidimensional scaling (MDS)}

Given the pairwise distance $d_{i j}$ between $n$ points and assuming that we don't know the exact coordinates of the points and how the distance is calculated, MDS (also known as Principal Coordinates Analysis [8]) tries to place these points in low dimensional space in such a way that the Euclidean distance between them is as close as possible to $d_{i j}$. Historically, the most significant achievement on MDS begins with Torgerson's work in 1952 [9]. Before then, Young and Householder [10] used the Euclidean distance as a metric of similarity measure. Let $X$ and $Y$ be metric spaces and $f: X \rightarrow Y$ an arbitrary map. The distortion of $f$ is defined by:

$$
\operatorname{dis} f=\sup _{a . b \in X}\left|d_{Y}(f(a), f(b))-d_{X}(a, b)\right| .
$$

The distance $d_{X}(a, b)$ between a pair of points in $X$ is mapped to the distance $d_{Y}(f(a), f(b))$ between the images of $a$ and $b$ under $f$ [11]. In our point cloud setting, where the shape $X$ is sampled at $N$ points $X=\left\{x_{1}, \ldots, x_{N}\right\}$, the distortion criteria will be:

$$
\sigma=\max _{i, j=1, \ldots, N}\left|d_{\mathbb{R}^{m}}\left(f\left(x_{i}\right), f\left(x_{j}\right)\right)-d_{X}\left(x_{i}, x_{j}\right)\right|
$$

In MDS literature, the function $\sigma$ which measures the distortion of distances is called stress. Historically $\sigma_{2}$ is used as the distortion criterion. Assume that $Z_{i}=f\left(x_{i}\right)$ is a matrix of canonical form coordinates and $d_{i j}(Z)=$ $d_{\mathbb{R}^{m}}\left(z_{i}, z_{j}\right)$, then:

$$
\sigma_{2}\left(Z ; D_{X}\right)=\sum_{i>j}\left|d_{i j}(Z)-d_{X}\left(x_{i}, x_{j}\right)\right|^{2} .
$$

Here $D_{X}$ is a matrix of geodesic distances and $d_{i j}(Z)$ is the Euclidean distance between the points on the canonical form. The minimization algorithms which minimize the stress function known as Multidimensional scaling. Historically MDS is classfied as a dimensionality reduction method. Scaling by Majorizing a COmplicated Function (SMACOF) is one of the well-known MDS algorithms for minimizing the stress function $\sigma_{2}\left(Z ; D_{X}\right)$ with respect to $Z$. This algorithm was proposed by De Leeuw [12]. This algorithm is the core of our study in reference [4]. Here we present a brief introduction on SMACOF. We refer the reader to [13] for an account. Before summarizing the SMACOF algorithm, we describe some relations and notations. Equation (3) can be written in matrix form:

$$
\begin{aligned}
\sigma_{2}\left(Z ; D_{X}\right)= & \operatorname{tr}\left(Z^{T} V Z\right)-2 \operatorname{tr}\left(Z^{T} B\left(Z ; D_{X}\right) Z\right) \\
& +\sum_{i>j} d_{X}^{2}\left(x_{i}, x_{j}\right)
\end{aligned}
$$

Here $V$ is a constant $N \times N$ matrix with elements:

$$
v_{i j}=\left\{\begin{array}{cc}
-1 & i \neq j \\
N-1 & i=j
\end{array}\right.
$$


and $B\left(Z ; D_{X}\right)$ is an $N \times N$ matrix with elements:

$b_{i j}\left(Z ; D_{X}\right)=\left\{\begin{array}{cc}-d_{X}\left(x_{i}, x_{j}\right) d_{i j}^{-1}(Z) & i \neq j \text { and } d_{i j}(Z) \neq 0 \\ 0 & i \neq j \quad \text { and } d_{i j}(Z)=0 \\ -\sum_{i \neq k} b_{i k} & i=j .\end{array}\right.$

Thus, the SMACOF algorithm can be summarized as:

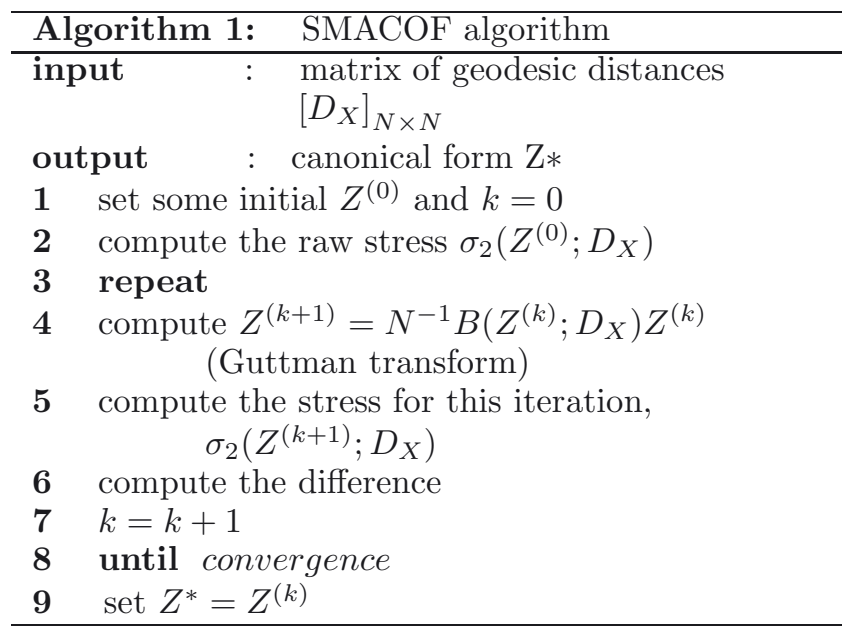

Step 6 of SMACOF algorithm contains findings for the difference in the stress values between the two previous iterations. If it is less than some predefined tolerance or if the maximum number of iterations has been reached, then the algorithm stops.

\subsubsection{Isometric feature mapping (ISOMAP)}

This technique described by Tenenbaum et al. [14] is the variant of MDS which uses graph distance (obtained by Dijkstra algorithm [15]) as an estimation of geodesic distance and applies MDS to lower the dimension of input data. The ISOMAP technique can be summerized as:

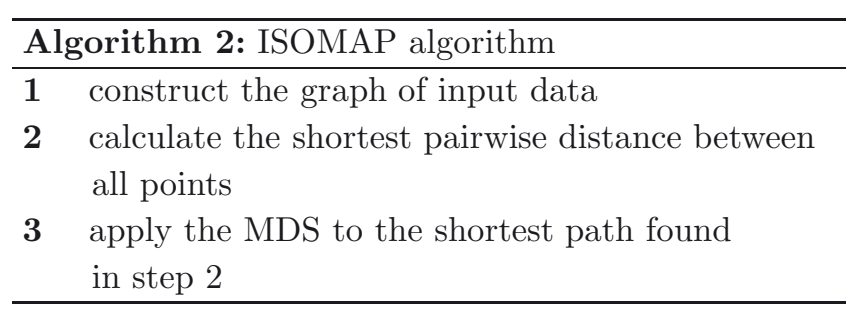

\subsubsection{Maximum variance unfolding (MVU)}

Weinberger and Saul [16] developed MVU algorithm (also known as Semidefinite Embedding) based on mapping the high dimensional data set into a low dimensional space that preserves the distance and angle between nearby input patterns. In MDS the pairwise Euclidean distance of input date sets was used as they were. In ISOMAP, Euclidean distance was replaced by geodesic distance. In MVU the transformation of distance is somehow more complicated than in MDS and ISOMAP. Distances are assumed to be preserved locally, while nonlocal distances are optimized in such a way that suitable embedding can be found. For instance, in 3D data sets the pairwise Euclidean distance is shorter than 2-dimensional embedding. Therefore MVU is considered to maximize the long distances while maintaining the shortest ones.

To this end, the aim of the MVU is to unfold data by maximizing pairwise distances, i.e.:

$$
\operatorname{Max} \sum_{i j}\left\|\vec{z}_{i}-\vec{z}_{j}\right\|^{2}
$$

subject to

$$
\forall(i, j) \in \text { edges } ; \ldots\left\|\vec{x}_{i}-\vec{x}_{j}\right\|^{2}=\left\|\vec{z}_{i}-\vec{z}_{j}\right\|^{2}
$$

and

$$
\sum_{i} \vec{z}_{i}=\overrightarrow{0}
$$

The latter constraint was put in place to eliminate translational degrees of freedom in the lower space by centering the output on the origin. The aforementioned optimization objective is a non-convex problem (multiple local minima) because it means maximizing a quadratic form subject to quadratic equality constraints. In reference [16] the authors propose a Semi definite Programming [17] technique by using dot products instead of squared distances. If $D$ denotes the square matrix of squared Euclidean distances, and $K$ the Gram matrices of $X$; i.e. $K_{i j}=x_{i} . x_{j}$, without going into detail, the MVU algorithm can be summarized as follows:

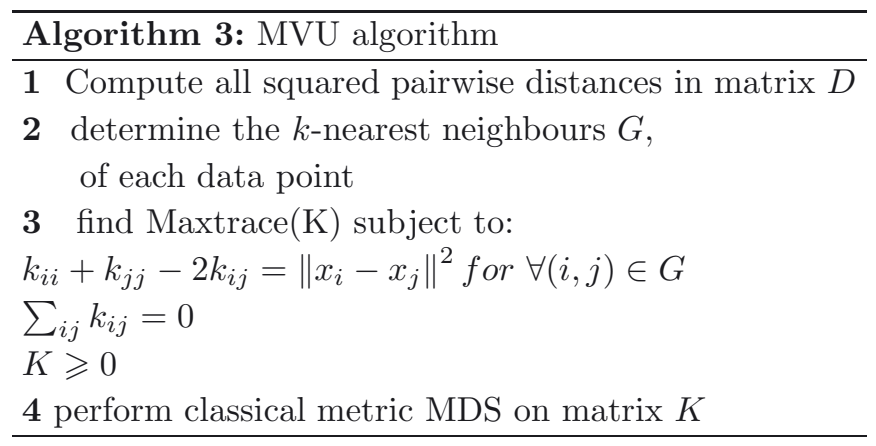

\subsubsection{Sammon's mapping}

The main weakness of MDS is that it tries to maintain large pairwise distances and does not retain the small ones [18]. Sammon' Mapping (SM) [19] tries to overcome MDS' weakness by weighting the contribution of each pair. To this end, SM minimizes the following stress function:

$$
E_{S M}=\frac{1}{\sum_{i j} d_{X}\left(x_{i}, x_{j}\right)} \sum_{\substack{i=1 \\ i<j}} \frac{\left(d_{X}\left(x_{i}, x_{j}\right)-d_{Z}\left(z_{i}, z_{j}\right)\right)^{2}}{d_{X}\left(x_{i}, x_{j}\right)}
$$

where $d$ is measured by Euclidean metrics. The minimization of Sammons's stress function can be performed using a pseudo-Newton optimization method. 


\subsubsection{Curvilinear component analysis (CCA)}

Originally developed by Demartines and Hérault [20], Curvilinear Component Analysis (CCA) is an improvement of Sammon's mapping. This technique combines some of the attitudes of SM and MDS along with artificial neural network strategies in order to map the higher dimensional data to lower dimensional space. At first, CCA processes a vector quantization step [21] as a way to reduce the data set size. Then like MDS, the authors defined a stress function in such a way as to preserve the interpoint distances during mapping. The CCA stress function closely resembles Sammon's stress function:

$E_{C C A}=\frac{1}{2} \sum_{i=1}^{N} \sum_{j=1}^{N}\left\{d_{X}\left(x_{i}, x_{j}\right)-d_{Z}\left(z_{i}, z_{j}\right)\right)^{2} F_{\lambda}\left(d_{Z}\left(z_{i}, z_{j}\right)\right\}$

while we would like to have $d\left(x_{i}, x_{j}\right)=d\left(z_{i}, z_{j}\right)$, this is not always possible without distortion, so they introduced a weighting function $F_{\lambda}$. The choice of $F_{\lambda}$ is based on the fact that preserving the short distances is more significant than the longer ones, because the long distances on the manifold have to be stretched to unfold the manifold. Thus, $F_{\lambda}$ was choosing as monotically decreasing function [21]. In order to minimize cost function, Demartines and Hérault [20] developed a novel variant of gradient descent techniques. We refer the reader to their original work for an account. In our study we didn't sampled the range data. Therefore, the vector quantization is considered an optional processing. Curvilinear Distance Analysis (CDA) developed by Lee et al. [22] is considered a variant of CCA which uses graph distance instead of Euclidean distance.

\subsection{Topology preserving techniques}

As depicted in the previous section dimensionality reduction can be reached by distance preservation. In this category numerous methods were discussed. While the comparative distances seem to give sufficient information on manifold, most distance functions make no distinction between manifold and its surrounding space. Topology preserving methods are another class of dimensionality reduction techniques that tend to preserve important structures of the data in the geometric structure of the mapping. One simple example of topology preserving maps is a Mercator projection of the earth into 2D space. While this kind of mapping gives invaluable visual information, distortion can't be prevented in some areas. In metrology, the topology gives the neighbourhood relationship between defect areas and the rest of the shape. The most problematic area in topology preserving techniques is how to represent a topology. All physical objects subjected to metrology are continuous. Unfortunately continuous topology representation is not always possible. This is why discrete representation is used by a 'lattice' (or grid). In this category we have selected the most well-known technique which we will summarize in the next section.

\subsubsection{Locally linear embedding (LLE)}

Locally linear embedding [23] is an eigenvector based technique (like PCA and MDS) where optimization doesn't involve local minima and iterative optimizations. It tries to preserve the local angles. LLE supposes that each point with its neighbors on the manifold lies on or close to a locally linear patch. Then it tries to characterize the local geometry of the patches by finding linear coefficients that reconstruct each point by using its $k$ nearest neighbors. Roweis and Saul [23] measured the reconstruction error by:

$$
\varepsilon(w)=\sum_{i}\left|x_{i}-\sum_{j} w_{i j} x_{j}\right|^{2}
$$

where $x_{j}$ is the $k$-nearest neighbors of $x_{i}$. $w_{i j}$ summarizes the contribution of the $j$ th data point to the $i$ th reconstruction and are found by optimizing equation (12) subject to $\sum_{j} w_{i j}=1$. The authors found optimal weights by using a least squares method. The final step of the algorithm is to reconstruct a representation $z_{i}$ of the $x_{i}$ in a low dimensional space. This was performed by minimizing the embedding cost function:

$$
\Phi(z)=\sum_{i}\left|z_{i}-\sum_{j} w_{i j} z_{j}\right|^{2} .
$$

The authors also proposed a sparse eigenvector problem in order to minimize the aforementioned cost function. We refer the reader to LLE's original paper for more details on the minimization technique.

The comparison and review of DR methods on Pattern classification and Data visualization can be found in references $[24,25]$.

\section{Experiment and results}

In the previous section we summarized some well-known NLDR techniques. In this section, the systematic comparison of the methods, along with their accuracy (minimum correspondence error) and performance in typical mechanical parts, will be investigated. To this end, we have categorized the very real engineering problems to four groups. Flexible parts with:

(1) zero Gaussian curvature with sharp edge (study case A);

(2) more complex shape with mostly zero Gaussian curvature (study case B);

(3) free-form high curvature (study case C);

(4) combination of both (study case D).

The aim of this study is to investigate the performance of NLDR methods on nonrigid parts from the viewpoint of metrology. To this end, all case studies (CAD-model \& range data) are considered to be intrinsically similar [2]. This means that all case studies considered are geometrically defectless. Figure 1 illustrates four case studies investigated for this study. The models were created by CATIA $^{\circledR}$ V5. Afterwards, a finite element analysis of the 


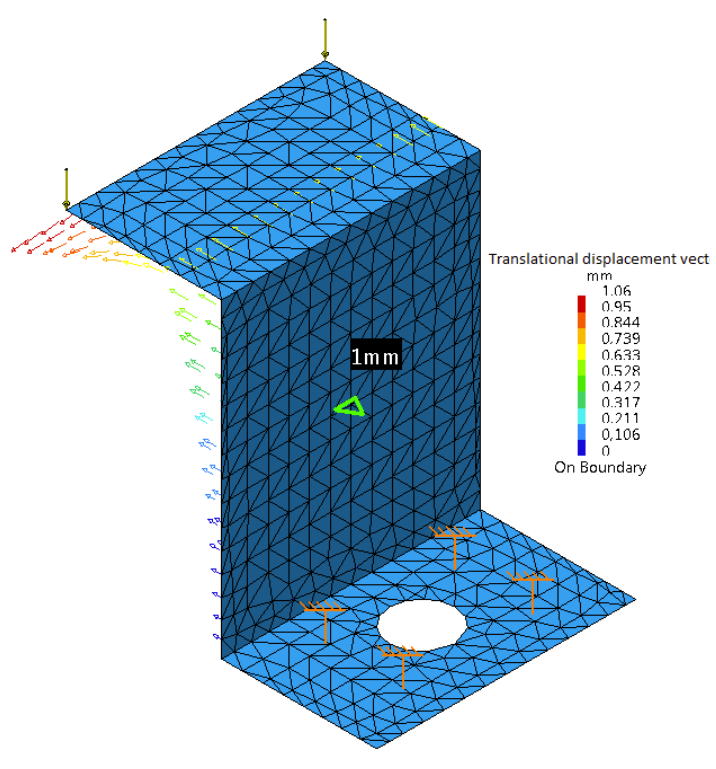

(A)

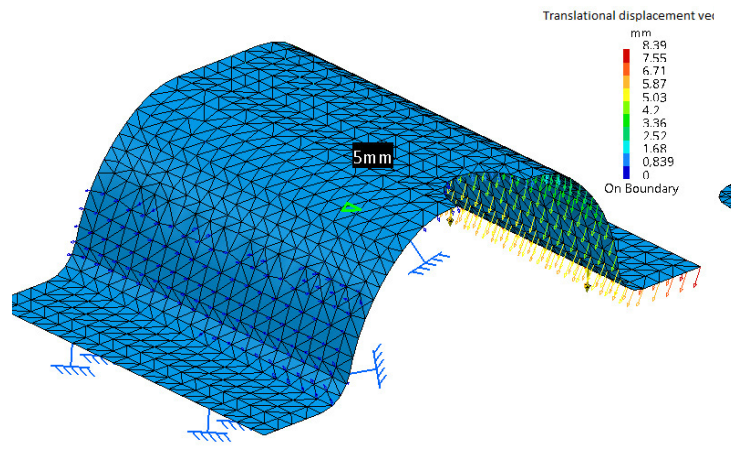

(C)

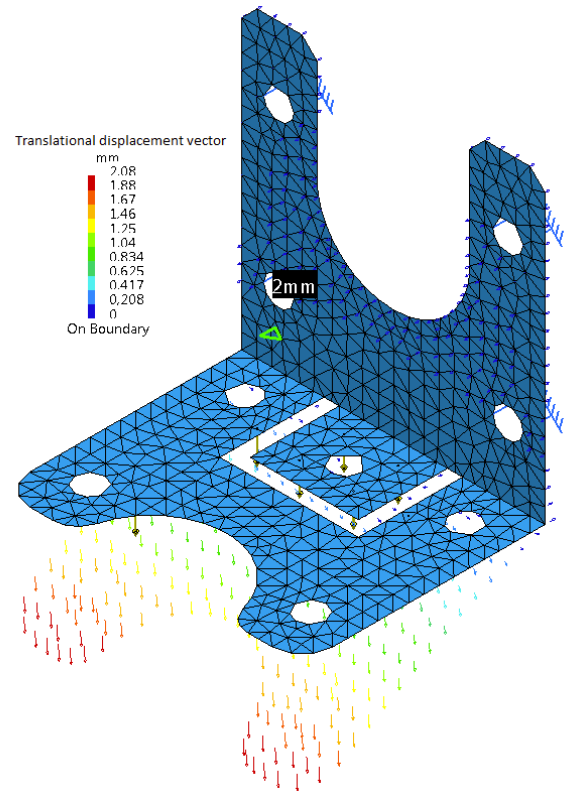

(B)

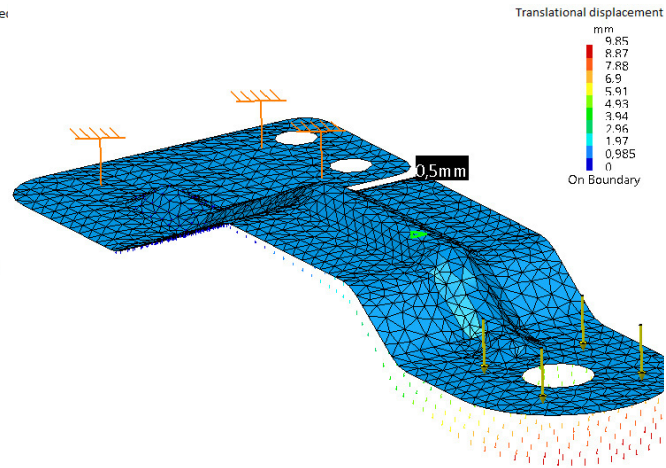

(D)

Fig. 1. Study cases.

model was performed to simulate the free-state range data. At this point, a displacement and/or a force were applied to the model to simulate spring back deformations. Then arbitrary translational and rotational displacements were added to the range data. In this way, the CAD-model and range data were simulated in different coordinate systems. Table 1 represents the geometric and mechanical properties of the case studies. In order to compare similarities between the CAD-model and range data after reducing the dimensionality, a Procrustes analysis was performed. Then the Euclidean distances between all corresponding points have been calculated. As an instance the performance study on the case study D is presented in Figure 2. All case studies were performed on an AMD Phenom(tm) II X4 B95 Processor $3.00 \mathrm{GHz}$ PC using a 64-bit operating system. Table 2 demonstrates the computational time for each NLDR algorithm. The results of the analysis as mean (Accuracy) and standard deviation (Precision) for all study cases were illustrated in Table 3 . The effect of registration error is considered to be equal for all case studies.
Table 1. Geometric and mechanical properties of case studies.

\begin{tabular}{ccccc}
\hline $\begin{array}{c}\text { Case } \\
\text { study }\end{array}$ & Material & $\begin{array}{c}\text { Thickness } \\
{[\mathrm{mm}]}\end{array}$ & $\begin{array}{c}\text { Dimension } \\
{[\mathrm{mm}]}\end{array}$ & \# of nodes \\
\hline A & Al-6061-T6 & 1.0 & $120 \times 120 \times 100$ & 496 \\
B & Al-6061-T6 & 2.0 & $100 \times 100 \times 80$ & 701 \\
C & Al-6061-T6 & 5.0 & $1600 \times 1000 \times 450$ & 996 \\
D & Al-6061-T6 & 0.5 & $340 \times 130 \times 50$ & 1322 \\
\hline
\end{tabular}

\section{Discussion}

According to the results of means and standard deviations, Table 4 illustrates the overall performance of dimensionality reduction methods for each study case. For free form high curvature parts (Study case C), a graph distance based ISOMAP perform better than other methods. This is something we already expected. In references [2-4] the authors used geodesics instead of graph distance as a similarity measure. However experiments shows that ISOMAP stands behind Sammon's nonlinear mapping as one of the computationally high casting methods. 

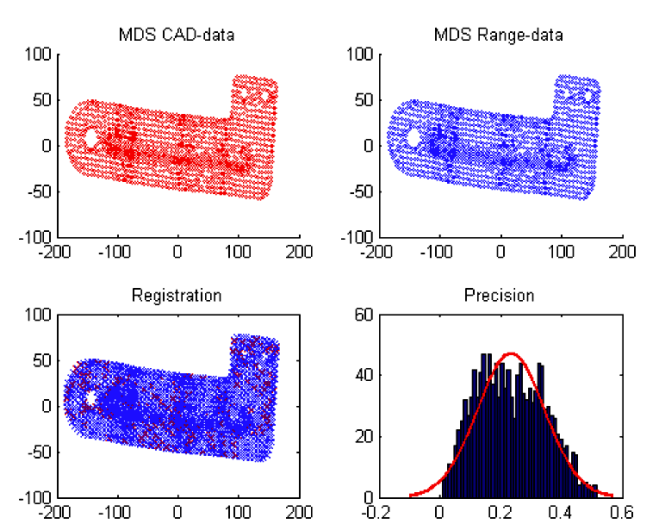

(a)
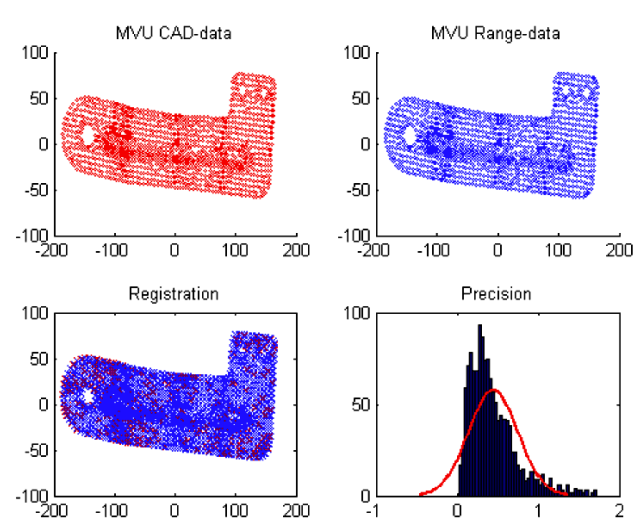

(c)
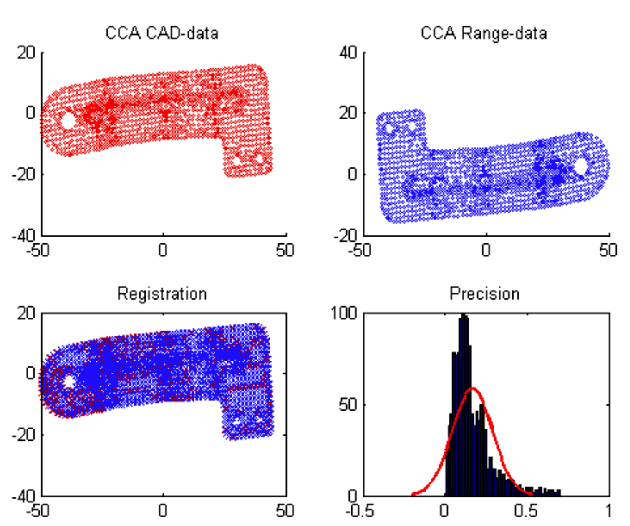

(e)
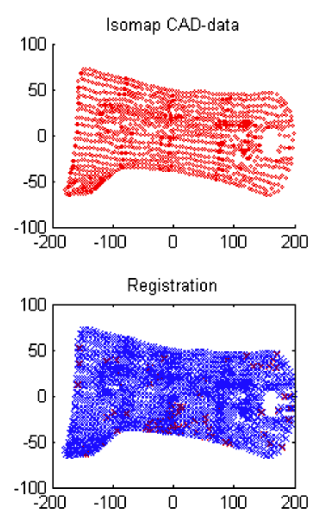

(b)
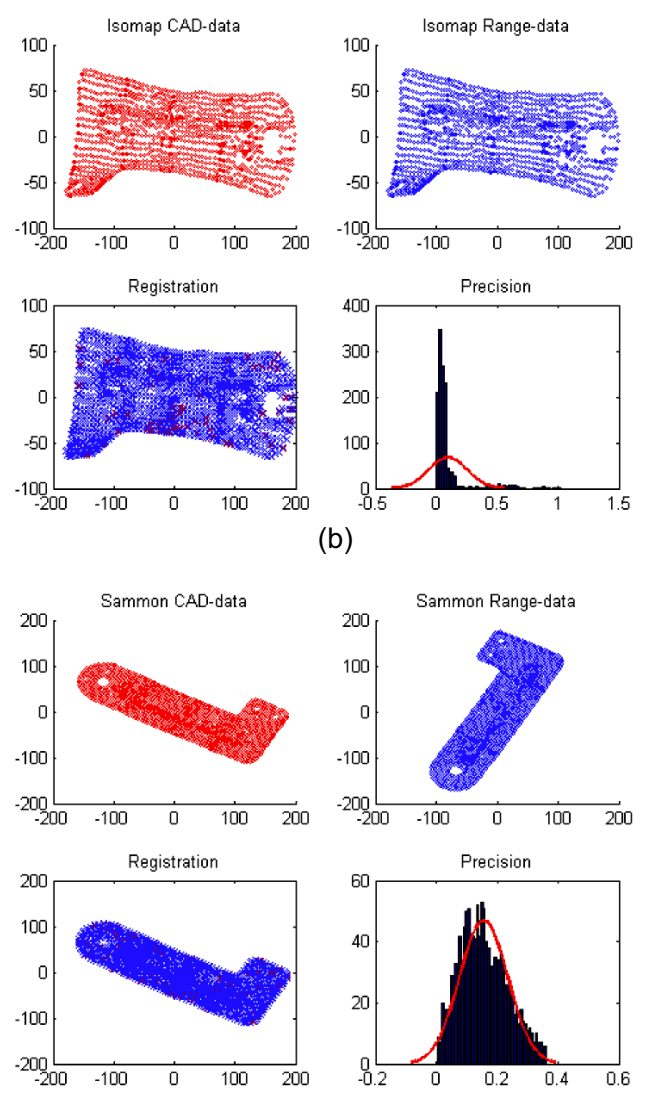

(d)
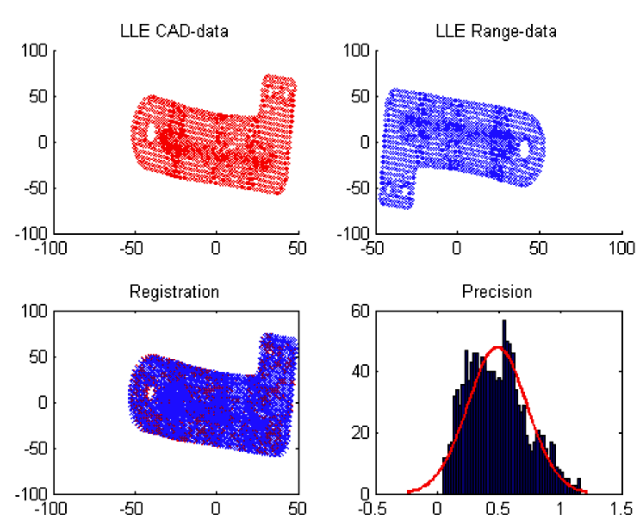

(f)

Fig. 2. 2D embedding of case study (D) using: (a) MDS; (b) ISOMAP; (c) MVU; (d) SM; (e) CCA; (f) LLE.

Classical MDS can be effectively used in simple parts with zero Gaussian curvature. On the other hand, classical MDS stands to be the fastest among the others. The performance of ISOMAP is notably worse than classical MDS in cases where parts have zero Gaussian curvature with sharp corners. The reason behind this phenomenon is the error of the geodesic/graph distance computation where the sharp bends occur. However, where the complexity increases (in the absence of sharp bends), ISOMAP offers more chances to achieve good precision. Although MVU uses the graph distance, it doesn't perform in the same manner as ISOMAP. Classical scaling cost functions used by Isomap retain the large geodesic/graph distances, while MVU focuses on keeping the local/small structure data.
Table 2. Computational time $[\mathrm{s}]$.

\begin{tabular}{ccccccc}
\hline $\begin{array}{c}\text { Case } \\
\text { study }\end{array}$ & MDS & ISOMAP & MVU & SM & CCA & LLE \\
\hline (A) & 0.371 & 15.596 & 0.930 & 16.493 & 1.050 & 0.789 \\
(B) & 0.782 & 33.202 & 3.073 & 40.840 & 2.236 & 1.097 \\
(C) & 1.096 & 72.249 & 4.086 & 106.172 & 3.788 & 2.346 \\
(D) & 1.912 & 140.833 & 6.004 & 297.410 & 5.345 & 4.525 \\
\hline
\end{tabular}

MVU should be avoided in the case of free form highly curved parts with large deformations where the curvature changes instantly.

Unlike classical MDS and MVU, Sammon's mapping can effectively handle all kinds of linear and nonlinear 
Table 3. Mean and standard deviation.

\begin{tabular}{cccccccc}
\hline $\begin{array}{c}\text { Case } \\
\text { study }\end{array}$ & & MDS & ISOMAP & MVU & SM & CCA & LLE \\
\hline \multirow{2}{*}{ (A) } & mean & 0.09 & 1.78 & 1.95 & 0.056 & 0.86 & 1.18 \\
& std & 0.06 & 1.42 & 0.99 & 0.03 & 0.44 & 0.78 \\
\hline \multirow{2}{*}{ (B) } & mean & 0.29 & 0.30 & 0.14 & 0.18 & 0.08 & 3.84 \\
& std & 0.16 & 0.17 & 0.10 & 0.07 & 0.06 & 2.38 \\
\hline \multirow{2}{*}{ (C) } & mean & 0.61 & 0.38 & 11.56 & 0.47 & 0.44 & 12.15 \\
& std & 0.26 & 0.23 & 10.24 & 0.19 & 6.04 & 8.52 \\
\hline \multirow{2}{*}{ (D) } & mean & 0.23 & 0.10 & 0.44 & 0.16 & 0.17 & 0.50 \\
& std & 0.11 & 0.16 & 0.30 & 0.08 & 0.12 & 0.24 \\
\hline
\end{tabular}

Table 4. Overall performance of NLDR methods in metrology.

\begin{tabular}{lcccc}
\hline & Study & Study & Study & Study \\
& case A & case B & case C & case D \\
\hline MDS & $\star \star \star \star$ & $\star \star$ & $\star \star$ & $\star \star$ \\
ISOMAP & $\star \star$ & $\star$ & $\star \star \star \star$ & $\star \star \star \star$ \\
MVU & $\star$ & $\star \star \star$ & $\star$ & $\star \star$ \\
SM & $\star \star \star \star$ & $\star \star \star$ & $\star \star \star$ & $\star \star \star$ \\
CCA & $\star \star \star$ & $\star \star \star \star$ & $\star \star \star$ & $\star \star \star$ \\
LLE & $\star \star$ & $\star$ & $\star$ & $\star$ \\
\hline
\end{tabular}

manifolds. While its global convergence is not always guaranteed it is also the most time-consuming NLDR technique.

By comparison, CCA proves to be much more flexible and can handle most linear and nonlinear data sets mostly because it gives the user the possibility of choosing the weighting function $F_{\lambda}$. In spite of the fact that CCA's cost function is mostly like Sammon's mapping, its convergence is faster.

The results of our experiments show that in spite of LLE's simplicity (there are only two parameters to be set); this topology preserving technique doesn't outperform the distance preserving techniques. In fact, the performance of LLE is somehow disappointing for the majority of realworld parts. LLE suffers from a fundamental weakness in its cost function [26].

\section{Conclusion}

With references [2-4] the authors have pioneered the concept of dimensionality reduction methods in $3 \mathrm{D}$ geometric metrology. In this paper we presented a review and systematic comparison between NLDR methods in order to evaluate their performance for applications on the metrology of flexible parts. We showed that even though these techniques may give acceptable results by artificial data on some fields like pattern recognition and machine learning, their performance cannot be extended to real engineering problems such as geometric metrology where high accuracy is needed. In spite of their undeniable performance for the metrology of flexible parts, special attention should be paid to each case for selecting the particular nonlinear dimensionality reduction technique.
Acknowledgements. This research is partially supported by the National Sciences and Engineering Research Council (NSERC). We also appreciate the authors of different codes in NLDR methods.

\section{Nomenclature}

\begin{tabular}{|ll|}
\hline$\sigma$ & stress (loss function) \\
$\left(X, d_{X}\right)$ & metric space where $d$ is a metric on $X$ \\
$D_{X}$ & $\begin{array}{l}\text { symmetric matrix of pair-wise geodesic } \\
\text { distances. (For } n \text { points, it requires } \\
n(n-1) / 2 \text { calculations.) }\end{array}$ \\
& $\begin{array}{l}\text { distance between a pair of points on } X \\
d_{X}(a, b)\end{array}$ \\
$\mathbb{R}^{n}$ & $n$-dimensional Euclidean space \\
$V^{T}$ & Transpose of matrix $V$ \\
$X, Y$ & surface \\
$Y_{M}$ & space $Y$ sampled by $M$ points \\
\hline
\end{tabular}

\section{References}

1. G.N. Abenhaim, A. Desrochers, A. Tahan, Nonrigid parts' specification and inspection methods: notions, challenges, and recent advancements, Int. J. Adv. Manuf. Technol. 63, 741-752 (2012)

2. H. Radvar-Esfahlan, S.A. Tahan, Nonrigid geometric inspection using intrinsic geometry, Proceedings of The Canadian Society for Mechanical Engineering Forum 2010, Victoria, British Columbia (2010)

3. H. Radvar-Esfahlan, S.A. Tahan, Nonrigid Geometric Metrology using Generalized Numerical Inspection Fixtures, Precis. Eng. 36, 1-9 (2011)

4. H. Radvar-Esfahlan, S.A. Tahan, Distance preserving dimensionality reduction methods and their applications in geometric inspection of nonrigid parts, 5th SASTECH Conference, Iran, 2011

5. H. Radvar-Esfahlan, S.-A. Tahan, Robust generalized numerical inspection fixture for the metrology of compliant mechanical parts, Int. J. Adv. Manuf. Technol. 70, 11011112 (2013)

6. I. Jolliffe, Principal Component Analysis (Wiley Online Library, 2005)

7. J.A. Lee, M. Verleysen, Nonlinear Dimensionality Reduction (Springer, 2007)

8. J.C. Gower, Some distance properties of latent root and vector methods used in multivariate analysis, Biometrika 53, 325-338 (1966)

9. W.S. Torgerson, Multidimensional scaling: I. Theory and method, Psychometrika 17, 401-419 (1952)

10. G. Young, A.S. Householder, Discussion of a set of points in terms of their mutual distances, Psychometrika 3, 19-22 (1938)

11. D. Burago, Y. Burago, S. Ivanov, A course in metric geometry (American Mathematical Society, 2001)

12. J. De Leeuw, Applications of convex analysis to multidimensional scaling (Department of Statistics Papers, Department of Statistics, UCLA, 2005)

13. I. Borg, P.J.F. Groenen, Modern multidimensional scaling: Theory and applications (Springer Verlag, 2005) 
14. J.B. Tenenbaum, V. De Silva, J.C. Langford, A global geometric framework for nonlinear dimensionality reduction, Science 290, 2319-2323 (2000)

15. E. Dijkstra, A note on two problems in connexion with graphs, Numer. Math. 1, 269-271 (1959)

16. K.Q. Weinberger, L.K. Saul, Unsupervised learning of image manifolds by semidefinite programming, in Computer Vision and Pattern Recognition, 2004. CVPR 2004. Proceedings of the 2004 IEEE Computer Society Conference on, 2004, Vol. 2, pp. II-988-II-995

17. L. Vandenberghe, S. Boyd, Semidefinite programming, SIAM Rev. 38, 49-95 (1996)

18. L. Van der Maaten, E. Postma, H. Van den Herik, Dimensionality reduction: A comparative review, J. Mach. Learn. Res. 10, 1-41 (2009)

19. J.W. Sammon Jr., A nonlinear mapping for data structure analysis, Comput. IEEE Trans. 100, 401-409 (1969)

20. P. Demartines, J. Hérault, Curvilinear component analysis: A self-organizing neural network for nonlinear mapping of data sets, Neural Netw. IEEE Trans. 8, 148-154 (1997)
21. A. Gersho, R.M. Gray, Vector Quantization and Signal Compression (Kluwer Academic Pub., 1992), Vol. 159

22. J.A. Lee, A. Lendasse, M. Verleysen, Curvilinear distance analysis versus isomap, in Proceedings of ESANN, 2002, pp. $185-192$

23. S.T. Roweis, L.K. Saul, Nonlinear dimensionality reduction by locally linear embedding, Science 290, 2323-2326 (2000)

24. C.J. de Medeiros, J.A.F. Costa, L.A. Silva, A comparison of dimensionality reduction methods using topology preservation indexes, in Intelligent Data Engineering and Automated Learning-IDEAL 2011 (Springer, 2011), pp. $437-445$

25. H. Yin, Nonlinear dimensionality reduction and data visualization: a review, Int. J. Automat. Comput. 4, 294-303 (2007)

26. J. Chen, Y. Liu, Locally linear embedding: a survey, Artif. Intell. Rev. 36, 29-48 (2011) 